months of the age boundary for their service. Baseline questionnaire was utilised to confirm the eligibility of the case, current treatment and comorbidity. Additional data were collected on the adult service to whom they were referred. Consultants were also asked to evaluate the different aspects of an optimal transition.

The BPSU reported 135 cases with 64 returned questionnaires and 51 eligible ADHD cases in transition. The CAPSS reported on 115 cases with 47 returned questinnaires and 39 eligible ADHD cases in need of transition were identified.

Results from the surveillance period which ended in November 2016 are very exciting. In general they indicate poor transition processes for young people with less than $25 \%$ of clinicians holding a transition planning meeting or having a handover period and less than 50\% having the referral to an adult mental health service accepted.

Conclusions Both paediatricians and child psychiatrists are equally involved in the management of young children with ADHD. However, there are still gaps in the provision of transitions of ADHD adolescents across the lifespan in most UK regions.

National guidelines that aims to promote optimal transitions and reduce perceived barriers to transition of care for young people with ADHD out of children's to adult services should be established.

Funding acknowledgement This project was funded by the National Institute for Health Research - Health Services and Delivery Research programme (project ref: 14/21/52)

\section{G470 CO-OCCURRENCE AND CO-MORBIDITIES AMONG CHILDREN AND ADOLESCENTS WITH ADHD AND ASD IN A SCOTTISH LOCAL AUTHORITY}

MO Ogundele. Community Paediatrics Unit, NHS Fife, Glenwood Health Centre, Glenrothes, $U K$

\subsection{6/archdischild-2018-rcpch.458}

ADHD is the commonest childhood neuro-behavioural disorder, affecting $5 \%$ to $12 \%$ of school-age children and about $1 \%$ of children meet the criteria for ASD. Co-occurrence of ASD and ADHD is increasingly recognised after introduction of DSM-5. They both share difficulties with emotional control, attention and high levels of negative affect, with differing underlying motivational and behavioural tendencies.

Objectives We analysed the clinical characteristics of children and young people with ADHD and ASD audited within two Community Child Health clinics of a Scottish NHS Trust over a 12 month period.

Methods A retrospective review of all patients seen in the outpatient clinics between June 2016 and May 2017 within an NHS Region was carried out. ADHD was diagnosed using validated Swanson, Nolan, and Pelham -IV Questionnaire (SNAP-IV). Sleep problems were diagnosed empirically from detailed clinical history provided by the parents/carers/patients. ASD was diagnosed by a multidisciplinary approach involving detailed assessment individually by the Educational Psychologist, Clinical Psychologist, Speech and Language Therapist and the Community Paediatrician, followed by group discussion, using the ICD-10 checklist criteria to confirm or refute a Diagnosis.

Results ASD and ADHD constituted 13\% and 17\% of the clinic caseloads respectively. They presented with similar characteristics including male gender preponderance $(4.3: 1$ and $4.5: 1)$, proportion of new referrals $(20 \%$ and $18 \%)$ and discharges (22\% and 19\%) respectively. 19\% of ASD children had ADHD while 14\% of ADHD children had ASD. They had a similar range of co-morbidities but sleep, emotional problems and DCD were commoner among ADHD patients (52\%, 25\% and $19 \%$ vs $44 \%, 17 \%$ and $9 \%$ respectively). CAMHS and General Paediatricians were more commonly involved with ADHD patients $(33 \%$ and $14 \%$ vs $20 \%$ and 6\%) than ASD children.

ASD patients were equally distributed between young (59 years) and older school age (10-14 years) children (20\% and $18 \%$ respectively) while most ADHD patients were chiefly older (10-14 years). ADHD patients were on average 20 months older, attended more clinics (average 2.2 vs 1.6 ) and had higher number of co-morbidities (average 2.3 vs 1.6 ).

Table 1 summarises the clinical and epidemiological characteristics of children and adolescents with ADHD and ASD.

\section{Child Health Ethics and Law Special Interest Group}

\section{G471 MORAL DISTRESS, TRAUMA AND BURNOUT IN STAFF IN RELATION TO CHANGES IN PICU OUTCOMES, CHALLENGING CASES AND MEDIA INVOLVEMENT IN DISAGREEMENTS ABOUT END-OF-LIFE CARE}

${ }^{1} \mathrm{G}$ Colville, ${ }^{2} \mathrm{M}$ Rutt, ${ }^{3} \mathrm{Z}$ Berger, ${ }^{3} \mathrm{P}$ Titman, ${ }^{2,4} \mathrm{~J}$ Brierley. ${ }^{1}$ Paediatric Psychology Service, St George's University Hospitals NHS Foundation Trust, London, UK; ${ }^{2}$ Neonatal Intensive Care Unit, Great Ormond Street Hospital, London, UK; ${ }^{3}$ Paediatric Psychology Department, Great Ormond Street Hospital, London, UK; ${ }^{4}$ Department of Paediatric Bioethics, Great Ormond Street Hospital, London, UK

\subsection{6/archdischild-2018-rcpch.459}

Background Technological advances have decreased PICU mortality but increased the number of children surviving with disability or technologically-dependent. Death in PICU most frequently follows withdrawal of life-sustaining therapy (LST), increasingly after prolonged admissions for invasive organ support. Disagreements with families about cessation of life-sustaining therapy (LST) can be protracted, distressing for everyone, harmful to the child and ultimately require court adjudication. Little is known about the impact of this, or of that of social/other media campaigns when families decides to involve the press, as is increasingly the case

Method 50 staff (39 nurses; 9 doctors and 2 AHPs) were surveyed using the Moral Distress Scale-Revised (MD-R) questionnaire ${ }^{1}$; Trauma Screening Questionnaire (TSQ) and abbreviated Maslach Burnout Inventory (aMBI) together with several open-ended questions about their experiences and what they felt had been learned 3 months after the final court decision following one such high profile case.

Results Mean moral distress score (MDS-R) was 96, consistent with recent Canadian multi-centre PICU study and higher than average Adult ICU scores (57-83 in the literature). Specifically, in relation to the recent high profile case $15 \%$ scored in the clinically significant range for post- traumatic stress symptoms (TSQ); a significant number reported a number of sub-clinical symptoms; $68 \%$ reported being 'upset by reminders of the event' and 53\% reported 'heightened awareness of potential danger' to themselves and others at least 